\title{
Ergotherapeutische Behandlung psychisch kranker Menschen
}

- rgotherapeutische Behandlung psychisch kranker - Menschen an der Schnittstelle des stationären zum - ambulanten Setting - so lautete der Titel des ersten ergotherapeutischen Symposiums im Rahmen des jährlichen Kongresses der Deutschen Gesellschaft für Psychiatrie, Psychotherapie und Nervenheilkunde DGPPN in Berlin. In dieser und den nächsten Ausgaben erhalten Sie die Möglichkeit die einzelnen Referate nachzulesen.

Unter dem Gesamtkontext Ergotherapie am Übergang von der stationären zur ambulanten Behandlung und Versorgung beleuchten die einzelnen Artikel verschiedene fachlich und/oder sozialrechtliche Aspekte. Sie sollen Ihnen als psychiatrisch/ psychotherapeutisch tätigen Arzt oder Psychologen einen Einblick in die Arbeit und den sozialrechtlichen Bedingungen der im sozialpsychiatrischen Kontext tätigen Ergotherapeuten geben.

Dabei ist es kein Zufall, dass gerade Ergotherapeuten sich in einem Symposium mit den Themen Kooperation und Schnittstelle beschäftigen, denn durch ihren handlungsorientierten Ansatz sind Ergotherapeuten besonders von diesen Themen ,betroffen“.

Ohne Kooperation z.B. mit dem Arzt kann die ergotherapeutische Behandlung nur begrenzte Wirkung zeigen. Dies bezieht sich nicht nur auf den Aspekt der notwendigen ärztlichen Verordnung der Leistung Ergotherapie, sondern vor allem auch auf die interdisziplinäre Zusammenarbeit im Sinne einer für den Patienten positiv wirksamen Behandlung. Der Ergotherapeut benötigt Informationen über den Patienten vom Arzt, Psychologen, Sozialarbeiter u.a., um diese in seiner handlungsund alltagsorientierten Behandlung zu berücksichtigen.

Ebenso große Bedeutung hat für den Ergotherapeuten die Schnittstelle stationär zu ambulant. Unabhängig davon, ob der Patienten stationär in einer akuten Phase oder ambulant in einem postakuten Stadium behandelt wird, die Orientierung an der Lebenswelt des Patienten verpflichtet die Ergotherapie zum Blick auf die berufliche und soziale Situation des Patienten. Der Erfolg ergotherapeutischer Arbeit als Teil des sozialpsychiatrischen Behandlungsgefüges ist davon abhängig, dass es dem Patienten möglich ist, die in der ergotherapeutischen Behandlung erarbeiteten Kompetenzen und (Verhaltens-)
Veränderungen auch in seinen spezifischen Lebenskontext zu integrieren. Gelingt dies nicht, sind Brüche im Behandlungs- und Rehabilitationsverlauf, die den Patienten bis hin zum Rückfall destabilisieren können, vorprogrammiert.

Für den Behandlungs- und Rehabilitationserfolg ist es also existenziell, dass der Patient beim Übergang zwischen den Versorgungsstrukturen (ergo-) therapeutische Unterstützung erhält, denn wir alle wissen aus Erfahrung, dass viele Patienten im Anschluss an eine akute Erkrankung häufig noch damit überfordert sind, ihr Leben ohne Unterstützung zu meistern.

Die Artikel sollen Ihnen die Arbeit der Ergotherapie in den verschiedenen sozialpsychiatrischen Handlungsfeldern näher bringen, damit Kooperation leichter wird und Schnittstellen besser überwunden werden können.

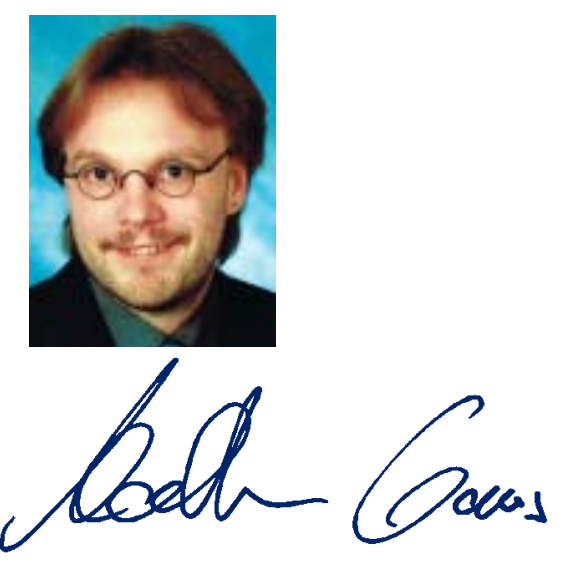

Mathias Gans, Tübingen

Deutscher Verband der Ergotherapeuten

Vorstandsmitglied 